\title{
RESEARCH PROGRESS ON RISK ACCEPTANCE CRITERIA OF LONG-DISTANCE OIL AND GAS TRANSPORTATION PIPELINE
}

\author{
WU ZONGZHI \\ China Academy of Safety Science and Technology \\ Beijing 100012, China \\ ZHANG SHENGZHU* \\ China Academy of Safety Science and Technology \\ Beijing 100012, China \\ E-mail: zhangshengzh5168@163.com \\ Received 14 November 2011 \\ Accepted 10 February 2012
}

\begin{abstract}
For acceptable risk and risk acceptance criteria, concepts and definition methods were proposed. Determination principles and application status including ALARP were investigated. Differences between long-distance oil/gas transportation pipeline and other industrial facilities in risk tendency, degree of risk control, environment pollution risk, and specific risk in pipeline shutdown were analyzed. Determination methods and standards of acceptable criteria of individual, social, property and environment risks were discussed. Individual and social risk standards of the pipeline were proposed according to China's actualities.
\end{abstract}

Keywords: long-distance oil and gas transportation pipeline, acceptable risk, acceptance criteria, standards

\section{油气长输管道风险接受准则研究进展 \\ 吴宗之 张圣柱 \\ 中国安全生产科学研究院, 北京 100012}

摘要: 在对可接受风险和风险接受准则的研究进展进行讨论的基础上, 归纳了其概念和界定方法。探讨了 ALARP 原则等风险可接受准则的确定原则, 以及各原则的应用现状。分析了油气长输管道与其他工业设 施在风险倾向、风险控制严格程度、环境污染风险, 以及管道特有的停输风险等方面存在的差异。讨论了 个人风险、社会风险、财产风险和环境风险的可接受准则确定方法及标准。结合中国实际情况, 提出了油 气长输管道的个人风险和社会风险标准。

关键词: 油气长输管道, 可接受风险, 接受准则, 标准

* Corresponding Author 


\section{1. 引言}

管道运输具有高效率、低成本且不易受运输周围环 境影响等优势, 已成为油气运输的首选方案。油品 和天然气具有易燃、易爆等特性, 并且管道储运工 艺复杂, 一旦出现故障容易引发火灾、爆炸等事 故, 导致严重人员伤亡、经济损失和环境污染。如 1999 年 6 月 10 日美国华盛顿州汽油管道爆炸事故造 成 3 人死亡、 8 人受伤、 1 处住宅及 1 座水处理厂受 到严重破坏，2000 年 8 月 19 日美国新墨西哥州天然 气管道爆炸事故造成 12 人死亡, 2010 年 5 月 2 日山 东胶州原油管道爆裂事故导致数百吨原油泄漏, 2011 年 5 月 31 日陕西铜川天然气管道闪爆事故迫使 上万人被紧急疏散等。尽管事故原因不同, 但这一 系列事故却反映出管道风险未能被有效控制的问 题。

自 20 世纪 70 年代起, 风险评价被广泛应用于油 气长输管道系统中, 通过估算长输管道系统的风险 值, 并以既定的风险可接受水平作为评价依据, 判 断风险值是否可以接受, 以此确定管道的安全水 平。确定合理的风险可接受水平是风险评价的关 键, 风险可接受水平过高会增加企业和社会成本, 过低则会导致风险被忽视, 给管道安全运行埋下隐 患。对于油气长输管道, 赵忠刚 ${ }^{1,2}$ 、秦岭 ${ }^{3}$ 、梁运 枚 ${ }^{4}$ 等从风险接受准则及其确定方法, 管道事故个 人可接受风险、社会可接受风险和环境可接受风险 等方面开展了研究, 但至今仍没有专门用于评价油 气管道事故可接受风险的统一标准, 另一方面, 风 险可接受水平的确定是基于历史统计数据之上的, 而我国对于长输管道事故的统计资料有限, 亟待开 展进一步研究。

目前我国油气长输干线管道己达 8 万公里, 事故 时有发生。随着境外油气资源的进一步落实, 西气 东输三线、四线等的构思与建设, 以及北油南运、 川气东送等规划, 未来几年将建设一批长输管道, 到 “十二五” 规划期末全国长输油气管道总里程将 超过 10 万公里 ${ }^{5}$ 。结合我国油气长输管道实际情 况, 借鉴相关国家成熟的风险可接受水平确定方 法, 探讨适用于我国的标准, 对于新建管道选线和 在役管道风险评价, 保障管道安全运行具有重要意 义。

\section{2. 可接受风险与风险接受准则}

20 世纪 60 年底末, 在核能、化工等领域相关学者开 始对风险的可接受性进行讨论。1968 年 Chauncey Starr 6提出了 “怎样安全才够安全” (How safe is safe enough)这一问题, 并根据 “揭示优先法” 得出了不
同风险的社会可接受性度量。1976 年 Lowrance $^{7}$ 在 其所著的 《Of Acceptable Risk: science and the determination of safety》一书中, 提出了 “只有认为 一个事物风险可接受时, 它才是安全的” 这一观 点。1981 年 Fischhoff ${ }^{8}$ 在所著的 《Acceptable Risk》 一书中对可接受风险进行了深入探讨, 认为可接受 风险问题是一个决策问题, 其可接受程度与对风险 的认识程度、甘愿冒险的程度、风险的可控程度、 灾害是否具有毁灭性以及恐惧心理等因素相关。 2001 年 $\mathrm{Marszal}^{9}$ 提出可接受风险是道德、法律和经 济上的一种平衡。英国健康和安全委员会(HSE)对可 接受风险的定义为任何可能会被风险影响的人, 为 了生活或工作的目的, 如果风险控制机制不变, 准 备接受的风险即为可接受风险。2000 年岑慧贤 ${ }^{10}$ 对 可接受风险的概念及其界定方法进行了研究, 认为 其是社会公众根据主观愿望对风险水平的接受程 度。

1988 年英国健康和安全委员会(HSE)发表了核电 站可容忍风险标准的研究报告《核电站的容许风 险》, 报告中指出一条 “地方不容许线” , 在该线 以上的风险被认为是不容许的风险，在同年的《可 容忍风险》报告中，HSE 认为低于 $10^{-6}$ 的死亡概率 是可以忽略的。挪威石油理事会将 “可接受性准 则”定义为 “用于表达工程的风险可接受性水 平”，它认为其 “指导方针” 是 “可接受性准则陈 述了人员伤亡、环境损害和财产、商业利益受损的 风险的特点”。风险接受准则可定义为在规定的时 间或系统的某一行为阶段内可接受的风险等级。它 是进行风险评价和采取风险控制措施的依据, 风险 接受准则可通过定量和定性的方式进行表示。

近年来, 国内一些学者对核电工程、建筑工程、 自然灾害等领域的可接受风险进行了研究并取得了 积极成果 $11,12,13$ 。对于油气长输管道可接受风险及 风险接受准则, 我国学者主要是在研究国外相关领 域可接受风险确定依据和方法的基础上，探讨了适 用于我国的风险接受准则, 对于可接受风险的实质 和符合我国实际的风险接受准则, 仍需开展深入研 究。

\section{3. 风险可接受准则的确定原则}

可接受风险水平过高会使风险被忽视，导致风险控 制措施不能有效实施。可接受风险水平也不是越小 越好, 在各领域中尤其是工程领域, 采取风险控制 措施就意味着人员和资金的投入，势必会使整个系 统的成本增加, 而且当风险降低到一定程度时, 风 险的进一步降低往往会以成本呈指数增加为代价。 应将风险确定在一个与经济社会发展相协调, 合 
理、可行的水平。风险可接受准则的制定需要满足 工程中的实际需要, 符合公认的行为标准, 并能从 自身活动和相关事故中得到经验 ${ }^{14,15}$ 。目前风险可 接受准则有以下几种:

\subsection{ALARP (As Low As Reasonably Practice)原 则。}

该原则通过容许上限和容许下限将风险划分为 3 个 区域(如图 1 所示)：风险水平超过容许上限即为不可 接受风险, 除特殊情况外该风险无论如何都不能被 接受, 相关活动必须停止; 风险水平低于容许下限 则风险被认为是可以接受的, 无需采取风险控制措 施; 风险水平处于容许上限和容许下限之间时, 可 采取合理可行的控制措施尽可能的来降低风险 16 , 17 。

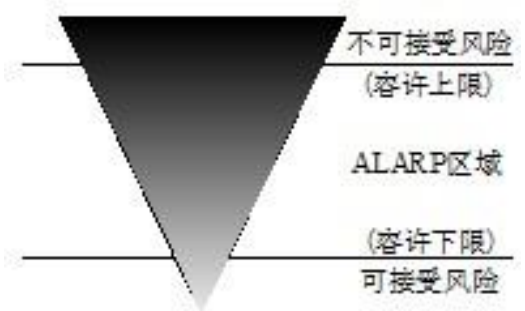

图 1 ALARP 原则

\subsection{GAMAB (Globalement Au Moins Aussi Bon) 原则。}

该原则指出新系统的风险与已经接受的现存系统的 风险相比较, 新系统的风险水平至少要与现存系统 的风险水平大致相当, 也称为比较原则。

\subsection{MEM (Minimum Endogenous Mortality) 原 则。}

该原则指出新活动所带来的危险不应比人们在日常 生活中所接触的其它活动的风险有明显增加, 一般 认为, 新活动所带来的风险增加量不应超过 $1 \%$ 。

目前国内外普遍采用 ALARP 原则, 即最低合理 可行原则, 对于处于不可接受区域的风险, 必须停 止相关活动或采取措施使风险降低到 ALARP 区 域, 对于处于 ALARP 区域的风险, 可采取合理可 行措施使之尽可能降低。
油气长输管道具有距离长、分布范围广的特点, 在 整条管道长度上具有不同的风险倾向, 而其他工业 设施主要集中在一个区域, 风险差异较小; 油气长 输管道在选线过程中, 迫于周围环境实际情况, 极 小部分管道可能会靠近中高密度人员场所或高敏感 区域，而其他新建工业设施在选址过程中对于风险 的控制则更加严格; 油气长输管道系统故障容易导 致泄漏、火灾、爆炸等事故，与其他工业设施事故 一样, 会导致人员伤亡和经济损失, 但由于长输管 道多采用高压力、大管径管道进行输送, 一旦发生 事故容易造成油品和天然气的大量泄漏, 环境污染 更为严重。另一方面，其他工业设施仅是生产一种 商品或提供一项服务, 而油气长输管道却是社会经 济和人民生活命脉，一旦发生较长时间停输将会导 致严重后果, 公众对停输风险厌恶度高。下面对油 气长输管道风险的生命风险( 个人风险和社会风险)、 财产风险和环境风险的可接受准则确定方法及标准 进行探讨。

\section{1. 生命风险可接受准则确定方法及标准}

\subsection{1. 个人风险}

个人风险是指既定区域内某一固定位置上的人员个 体死亡概率, 与人员的数量和人员是否处于此位置 无关，只与该位置的空间坐标有关。目前个人风险 可用 AFR、AI、风险矩阵等方法进行确定。

AFR (Annual Fatality Risk) 即年死亡风险, 指一 个人在一年内的死亡概率, 其计算一般通过划分网 格的方法来实现, 需要根据危险源的全部事故情 景、所有的天气等级、气象状况进行综合计算并累 加得到。

AI (Aggregated Inidcator)即聚合指数, 指单位国 民生产总值的平均死亡率, 对于油气长输管道可通 过式(1)进行定义:

$$
A I_{p}=N_{p} / G N P_{p} .
$$

式中: $N_{\mathrm{p}}$ 一油气长输管道行业死亡人数;

$$
G N P_{\mathrm{p}} \text { 一油气长输管道行业所占国民生产总 }
$$
值。

风险矩阵通过将事故发生的可能性和严重度相叠 加得到风险等级 ${ }^{18}$, 如表 1 所示:

\section{4. 风险可接受准则的确定方法及标准}


表 1 风险评价矩阵

\begin{tabular}{|c|c|c|c|c|c|}
\hline 事故后果 & & & 风险程度 & & \\
\hline 事故可能性 & $\begin{array}{c}\mathrm{V} \\
\text { (可忽略的) }\end{array}$ & $\begin{array}{c}\text { IV } \\
\text { (轻度的) }\end{array}$ & $\begin{array}{c}\text { III } \\
\text { (中度的) }\end{array}$ & $\begin{array}{c}\text { II } \\
\text { (严重的) }\end{array}$ & $\begin{array}{c}\text { I } \\
\text { (灾难的) }\end{array}$ \\
\hline $\mathrm{A}($ 频繁 $)$ & $\mathrm{M}$ & $\mathrm{M}$ & $\mathrm{H}$ & $\mathrm{H}$ & $\mathrm{H}$ \\
\hline B(很可能) & $\mathrm{S}$ & M & M & $\mathrm{H}$ & $\mathrm{H}$ \\
\hline C(有时) & $\mathrm{L}$ & S & M & $\mathrm{H}$ & $\mathrm{H}$ \\
\hline D(极少) & $\mathrm{L}$ & $\mathrm{L}$ & S & M & $\mathrm{H}$ \\
\hline $\mathrm{E}$ (不可能) & $\mathrm{L}$ & $\mathrm{L}$ & $\mathrm{S}$ & M & M \\
\hline
\end{tabular}

其中： $\mathrm{H}$ 类为 “不可接受” 风险； $\mathrm{M}$ 类为 “不希望” 的风险； $\mathrm{S}$ 类为 “有条件的可接受” 的风险; $\mathrm{L}$ 类为 “可接受” 的风险。

目前个人风险多用 AFR 表示, 英国、荷兰、澳 大利亚等国家将个人死亡率低于 $10^{-6}$ 每年作为可接 受风险标准, 高于 $10^{-5}$ 作为不可接受标准, $10^{-5}$ 至 $10^{-6}$ 之间的风险需加强监控和整改 ${ }^{19}$ 。我国尚无统一 标准，国家 “十五”科技攻关项目《城市公共安全
规划技术、方法和程序研究》成果 ${ }^{20,21}$, 所建议的 个人风险容许标准如表 2 所示。

参照国外研究机构的建议标准和《危险化学品重 大危险源监督管理暂行规定》, 结合油气长输管道 的特点, 本文提出了油气长输管道的个人风险标 准, 如表 3 所示。

表 2 建议的个人风险容许标准

\begin{tabular}{|c|c|c|c|}
\hline 应用对象 & 典型对象 & $\begin{array}{l}\text { 最大可容许风险/ } \\
\text { 每年 }\end{array}$ & 标准说明 \\
\hline $\begin{array}{c}\text { 高敏感或高密度 } \\
\text { 场所 }\end{array}$ & $\begin{array}{l}\text { 党政机关、军事禁区、军事管理区、 } \\
\text { 古迹、学校、医院、敬老院、居民 } \\
\text { 区、大型体育场馆、大型商场、影剧 } \\
\text { 院、大型宾馆饭店等 }\end{array}$ & $1 \times 10^{-6}$ & $\begin{array}{l}\text { 在高敏感或高密度场所不接受 } \\
1 \times 10^{-6} \text { 的个人风险。1 } 1 \times 10^{-6} \text { 每年的 } \\
\text { 个人风险等值线不应进入该区 } \\
\text { 域。 }\end{array}$ \\
\hline 中密度场所 & $\begin{array}{l}\text { 零星居民、办公场所、劳动密集型工 } \\
\text { 厂、小型商场(商店)、小型体育及文化 } \\
\text { 娱乐场所等 }\end{array}$ & $1 \times 10^{-5}$ & $\begin{array}{l}1 \times 10^{-5} \text { 每年的个人风险等值线不 } \\
\text { 应进入该区域。 }\end{array}$ \\
\hline 低密度场所 & 技术密集型工厂、公园、广场 & $1 \times 10^{-4}$ & $\begin{array}{l}1 \times 10^{-4} \text { 每年的个人风险等值线不 } \\
\text { 应进入该区域。 }\end{array}$ \\
\hline 企业内部 & 企业内部 & $1 \times 10^{-3}$ & $\begin{array}{l}\text { 厂区内不应出现 } 1 \times 10^{-3} \text { 每年的个 } \\
\text { 人风险等值线。 }\end{array}$ \\
\hline
\end{tabular}

表 3 建议的油气长输管道个人风险标准

\begin{tabular}{|c|c|c|}
\hline 对象类型 & 典型对象 & $\begin{array}{c}\text { 最大可容许风险 } \\
\text { /每年 }\end{array}$ \\
\hline $\begin{array}{l}\text { 1. 高敏感场所 } \\
\text { 2. 重要目标 } \\
\text { 3. 特殊高密度场所 }\end{array}$ & $\begin{array}{l}\text { 1. 如学校、医院、幼儿园、养老院等 } \\
\text { 2. 如党政机关、军事管理区、文物保护单位等 } \\
\text { 3. 如大型体育场、大型交通枢纽等 }\end{array}$ & $3 \times 10^{-7}$ \\
\hline $\begin{array}{l}\text { 1. 居住类高密度场所 } \\
\text { 2. 公众聚集类高密度场所 }\end{array}$ & $\begin{array}{l}\text { 1. 如居民区、宾馆、度假村等 } \\
\text { 2. 如办公场所、商场、饭店、娱乐场所等 }\end{array}$ & $1 \times 10^{-6}$ \\
\hline 中密度场所 & $\begin{array}{l}\text { 如零星居民、办公场所、劳动密集型工厂、小型商场 } \\
\text { (商店)、小型体育及文化娱乐场所等 }\end{array}$ & $5 \times 10^{-6}$ \\
\hline 低密度场所 & 如技术密集型工厂、公园、广场等 & $5 \times 10^{-5}$ \\
\hline
\end{tabular}




\subsection{2. 社会风险}

社会风险是跟人员密度相关的函数, 指的是能够引 起大于等于 $\mathrm{N}$ 人死亡的事故累积频率 $\mathrm{F}$, 代表单位 时间(年)内的死亡人数, 与空间位置无关, 只受环境 人口密度的影响。目前社会风险可用 FN 曲线、

PLL、VIIH 等方法进行确定。

FN 曲线是以死亡人数( $N$ )对应各种事件后果发生 频率累加值 $(\mathrm{F})$ 作图的分布图形。FN 曲线将整个风 险区域划分为三部分, 包括不可容许区、ALARP 区 和可容许区, 根据实际得到危险源的社会风险曲线 落入的区域, 采取不同的风险控制措施。

PLL (Potential Loss of Life) 即潜在死亡人数, 表 示一个区域内可能因事故导致的全部死亡人数, 可 通过式(2)进行定义:

$$
P L L=\sum_{i=1}^{n} I R_{i} \cdot d_{i} .
$$

式中: $I R_{\mathrm{i}}$ 一第 $\mathrm{i}$ 个网格的个人风险; $d_{\mathrm{i}}$ 一第 $\mathrm{i}$ 个网格的人口数量;

$n$ 一网格的总数目。

VIIH (Value Injuries and Ill Health) 即受伤和不健 康值, 它假设一个人的死亡与一定数量的人员受伤 或健康损害相当, 从而综合考虑事故造成的人员受 伤和健康损害等影响, 解决了人员死亡以外的风险 量化问题。

目前社会风险多用 FN 曲线表示, 荷兰、英国、 香港等国家和地区的社会风险推荐标准如图 2 所 示, 其中, 荷兰风险标准最为严格, 英国风险标准 主要针对现有危险设施, 标准相对较低, 香港风险 标准介于两者之间。

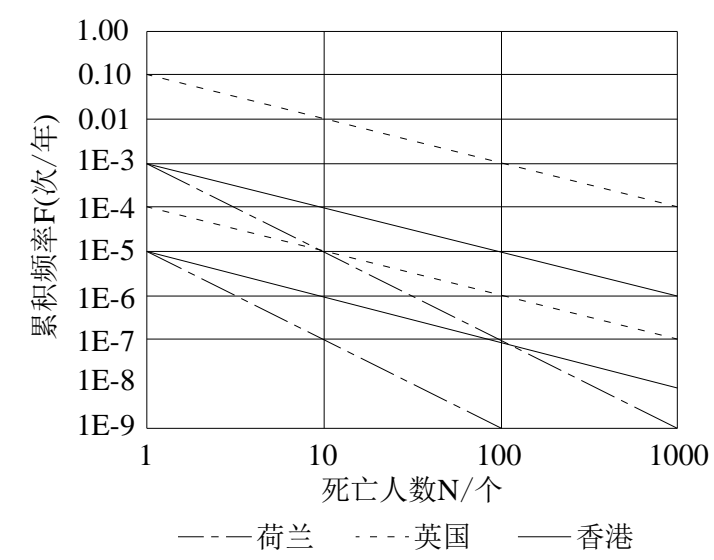

图 2 社会风险可接受标准
油气长输管道路线长、范围广, 在管道新建过程 中, 征地是最重要的问题, 标准过高会增加土地的 占用量, 致使建设成本过高, 不利于长输管道的发 展。另一方面, 与发达国家相比, 中国在油气长输 管道的技术、设备和安全管理等方面还存在差距, 达不到其安全运行水平, 需要逐步完善、提高。本 文建议采用香港的社会风险标准作为油气长输管道 的可容许社会风险标准(如图 2 所示)。

\section{2. 财产风险可接受准则确定方法及标准}

财产风险一般指整条管道或特定一段的总风险。类 似于 FN 曲线, 财产风险可接受准则可通过 PL 曲线 进行表示。 $\mathrm{P}$ 为财产损失累积频率, $\mathrm{L}$ 为财产损失 值, PL 曲线将整个区域划分为两部分, 曲线以上为 不可接受风险区，应采取措施进行控制，以下为可 接受风险区, 可不采取控制措施。秦岭等 ${ }^{3}$ 提出的 财产风险可接受标准如图 3 所示, 各概率的意义如 表 4 所示。

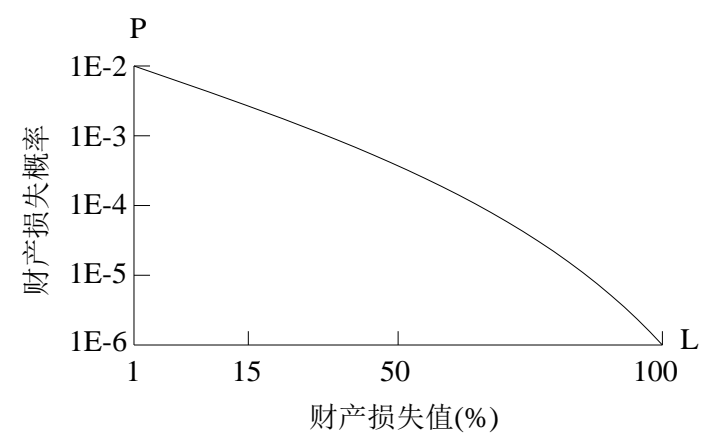

图 3 财产风险可接受标准

表 4 各概率所代表意义

\begin{tabular}{cl}
\hline 概率等级 & \multicolumn{1}{c}{ 意义 } \\
\hline $10^{-6}$ & 事故后果严重, 且无事先征兆 \\
$10^{-5}$ & 后果严重但事故发生前发出警告 \\
& 事故后果严重, 人员能够逃离, 财产损 \\
$10^{-4}$ & 失率约为 $50 \%$ \\
$10^{-3}$ & 后果不严重, 财产损失率约为 $10 \%$ \\
$10^{-2}$ & 损失很小, 如 $1 \%$ \\
\hline
\end{tabular}

\section{3. 环境风险可接受准则确定方法及标准}

环境破坏主要是因油品和气体泄漏造成的。可通过 环境破坏程度和可接受的频率确定环境风险可接受 
准则, 秦岭等 ${ }^{3}$ 提出的环境风险可接受标准如表 5 所 示。

表 5 环境风险可接受标准

\begin{tabular}{ccc}
\hline 环境破坏等级 & 平均恢复期(a) & 可接受频率 \\
\hline 较小 & 0.5 & $1 \times 10^{-1}$ \\
中等 & 2 & $2.5 \times 10^{-2}$ \\
重大 & 5 & $1 \times 10^{-2}$ \\
严重 & 20 & $2.5 \times 10^{-3}$ \\
\hline
\end{tabular}

现阶段, 风险可接受准则的确定主要是基于定量 风险计算之上的, 个人风险多用 AFR 表示, 社会风 险多用 FN 曲线表示, 财产风险可用 PL 曲线表示, 环境风险可用可接受频率与平均恢复期进行表示, 既有通用标准, 也有仅用于油气长输管道的标准。

标准的合理性与基础数据的丰富程度和可信性密 切相关, 目前, 标准主要来源于西方发达国家, 是 在对大量真实数据进行统计分析基础上确定的, 在 这些国家已被证明具有可操作性。与西方发达国家 相比, 我国在经济、社会发展状况以及自然环境方 面存在较大差异, 另一方面, 我国对相关数据的统 计滞后, 且完整性较低, 仍需对风险可接受准则的 确定方法和适用于我国的标准进行研究。

在借鉴工业设施风险标准的基础上, 本文提出了 油气长输管道的个人风险标准和社会风险标准与学 者共同探讨。公众对停输风险的厌恶度高, 且油气 长输管道泄漏事故造成的环境污染一般较为严重, 有待根据我国实际情况确定合理的停输和环境风险 标准。

\section{5. 建议}

风险接受准则研究是油气长输管道风险研究的关键 方面, 合理的风险标准是指导油气长输管道系统风 险控制的重要依据。针对存在的问题, 对今后的油 气长输管道风险接受准则研究提出以下建议:

(1) 目前我国在用的相关风险接受准则及标准均 来源于西方发达国家，准则的表述形式和标准的高 低是否适用于我国仍需讨论。建议政府安全生产主 管部门在研究的基础上提出我国的油气长输管道生 命、环境等风险标准。

(2) 政府安全生产主管部门和管道运营商共同建 立管道事故数据库, 详细记录我国境内不同区域的 管道失效数据, 确保所记录数据的规范性和通用 性, 为建立在概率统计基础上的风险接受准则的合 理确定提供依据。
(3) 受技术水平和管理水平的影响, 油气长输管 道风险可接受准则是动态变化的, 需要根据实际情 况对标准适时做出调整, 我国幅员辽阔、管道所经 过区域地质条件复杂多样，可探讨适用于不同地段 的差异性标准。

\section{致谢}

文章得到了国家 “863” 计划项目 (2009AA11Z211) 的资助。

\section{参考文献}

1. Z. Z. Gang, Y. A. Lin, Z. X. Fen, Research progress of the acceptability of the risk assessment of oil and gas pipelines, Technology Supervision in Petroleum Industry 5 (2005) 94-98.

赵忠刚, 姚安林, 赵学芬, 油气管道可接受性风险评估 的研究进展 [J], 石油工业技术监督, 2005, 5: 94-98.

2. Z. Z. Gang, Y. A. Lin, L. Y. Lv, L. D. Quan, Defining acceptable risk standard values of oil and gas pipeline, Journal of Southwest Petroleum University(Science \& Technology Edition) 30(2) (2008) 147-150.

赵忠刚, 姚安林, 李又绿, 李大全, 油气管道可接受风险 标准值的界定研究 [J], 西南石油大学学报(自然科学 版), 2008, 30(2): 147-150.

3. Q. Ling, C. L. Qiong, C. Kang, Acceptance criterion on risk in oil and gas pipeline, Natural Gas And Oil 25(2) (2007) 15-17.

秦岭, 陈利琼, 陈康, 油气管道风险接受准则 $[\mathrm{J}]$, 天然气 与石油, 2007, 25(2): 15-17.

4. L. Y. Mei, L. Hui, Y. Wei, Study progress in risk acceptance criteria of long-distance natural gas transmission pipeline, Oil \& Gas Storage And Transportation 28(10) (2009) 6-9.

梁运枚, 骆晖, 杨伟, 天然气长输管道风险接受准则的 研究进展 [J], 油气储运, 2009, 28(10): 6-9.

5. 华建敏. 十二五末我长输油气管道总里程超 10 万公里 [EB/OL].

2010-09-25 http://news.163.com/10/0925/20/6HF1Q16500014JB6.ht $\mathrm{ml}$

6. S. Chauncey, Social benefit versus technological risk, Science, 165(3899) (1969) 1232-1238.

7. Lowrance (eds.), Of acceptable risk: science and the determination of safety. (William Kaufmann Inc., California, 1976)

8. Fischhoff B, Lichtenstein S, Slovic P, et al. (eds.), Acceptable Risk. (Cambridge University Press, New York, 1981)

9. Marszal E M, Tolerable risk guidelines, Isa Transactions, 40(4) (2001) 391-399

10. C. H. Xian, F. H. Yang, W. Q. He, Concept of acceptable risk, Chongqing Environment Science 22(3) (2000) 1819. 
岑慧贤, 房怀阳, 吴群河, 可接受风险的界定方法探讨 [J], 重庆环境科学, 2000, 22(3): 18-19.

11. S. Z. Gang, Z. Z. Yi, X. Lan, Study of risk acceptance of nuclear power, Chinese Journal of Nuclear Science and Engineering 22(3) (2002) 193-198.

时振刚, 张作义, 薛澜, 核能风险接受性研究 [J], 核科学 与工程, 2002, 22(3): 193-198.

12. G. Z. Lin, J. Z. Ke, L. X. Hui, Study on the risk acceptance criteria in construction, J. Xi'an Univ. of Arch. \& Tech.(Natural Science Edition) 40(1) (2008) 76-79.

郭章林, 贾增科, 李晓慧, 建筑工程施工风险接受准则 研究 [J], 西安建筑科技大学学报(自然科学版), 2008, 40(1): 76-79.

13. S. Z. Hai, L. X. Lin, Acceptable risk and disaster research, Progress In Geography 29(1) (2010) 23-30.

尚志海, 刘希林, 可接受风险与灾害研究 [J], 地理科学 进展, 2010, 29(1): 23-30.

14. L. B. Yan, Study on criteria of acceptable Risk, master thesis, Zhenjiang: Jiangsu University, (2010), pp.5-23. 李宝岩, 可接受风险标准研究, 江苏大学硕士学位论文 [D], 江苏大学, 2010, 5-23.

15. Y. Fei, T. Tao, Research and development of safety technology in signaling system of rail transit, China Safety Science Journal 15(6) (2005) 94-99.

燕飞, 唐涛. 轨道交通信号系统安全技术的发展和研究 现状[J]. 中国安全科学学报, 2005, 15(6): 94-99

16. EN50126, Railway applications-The specification and demonstration of Reliability, Availability, Maintainability and Safety (RAMS) [S], 1999

17. Qingdao Safety Engineering Research Institute of China Petroleum \& Chemical Corporation (eds.), A guidance for quantitative risk assessment in the petrochemical plant. (China Petrochemical Press, Beijing, 2007)

中国石油化工股份有限公司青岛安全工程研究院, 石 化装置定量风险评估指南[M], 北京: 中国石化出版社, 2007.

18. W. Z. Zhi, A preliminary discussion on establishment of the system of industrial accident risk management in China, China Safety Science Journal 15(S) (1995) 183186.

吴宗之, 建立我国工业事故风险管理制度的探讨 $[\mathrm{J}]$, 中 国安全科学学报, 1995, 5(S): 183-186.

19. W. Z. Zhi, Study on methods and contents for land use safety planning, Journal of Safety and Environment 4(6) (2004) 86-90.

吴宗之, 城市土地使用安全规划的方法与内容探讨 [J], 安全与环境学报, 2004, 4(6): 86-90.

20. China Academy of Safety Science and Technology, Urban public safety planning techniques, methods and procedures, National scientific and technological research report of the tenth five-year of China, 2004.

中国安全生产科学研究院, 城市公共安全规划技术、 方法与程序研究 $[R]$, “十五” 国家科技攻关课题研究 报告, 2004.
21. W. Z. Zhi, D. Y. Quan, W. L. Jun, et al, Quantitative area risk assessment method and its application in land use safety planning for major hazard installation, Engineering Science 8(4) (2006) 46-49.

吴宗之, 多英全, 魏利军等, 区域定量风险评价方法及 其在城市重大危险源安全规划中的应用[J], 中国工程 科学, 2006, 8(4): 46-49. 